\title{
The Origin of Nonmagnetic Kramers Doublets in the Ground State of Dysprosium Triangles: Evidence for a Toroidal Magnetic Moment**
}

\author{
Liviu F. Chibotaru,* Liviu Ungur, and Alessandro Soncini
}

The design of single-molecule magnets (SMMs) based on the phenomenon of blocking of magnetization at low temperature $^{[1-6]}$ has become a hot area of research due to the potential applications of such compounds in new storage and information-processing technologies. ${ }^{[7-10]}$ Most of the SMMs synthesized so far are polynuclear transition metal complexes at their strong exchange limit, ${ }^{[11]}$ where the exchange splitting is much larger than the zero-field splitting on individual metal sites. An SMM effect is obtained in the case of axial zero-field splitting, $D S_{\mathrm{z}}{ }^{2}$, where $S_{\mathrm{z}}$ is the projection of the total spin $S$ on the symmetry axis of the cluster, with $D<0$. In order to have a large barrier for reversal of magnetization, $|D| S^{2}$, these complexes should possess a large $S$ and large magnetic anisotropy projected on the ground exchange multiplet $(D)$.

At the opposite limit of weak exchange coupling the zerofield splitting on metal sites is much larger than the exchange splitting in the complex. ${ }^{[1]}$ This is always the case for complexes containing lanthanides and actinides and is often so for transition metal complexes containing cobalt ions or second- and third-row transition metal ions. The high anisotropy on lanthanide ions has prompted investigations of the effect of their incorporation on the SMM performance ${ }^{[12-17]}$ as both the ionic anisotropy and exchange interaction in these complexes can contribute to the height of the barrier of reversal of magnetization. The first mechanism alone is responsible for the SMM effect in the mononuclear bis(phthalocyaninato)holmium anion. ${ }^{[13]} \mathrm{A}$ pure exchange contribution to the barrier has been predicted for some complexes with axial symmetry involving heptacyanomolybdenum(III). ${ }^{[18]}$ Generally, however, the origin of SMM behavior of complexes at the weak exchange limit is difficult to elucidate.

Three compounds containing a triangle of $\mathrm{Dy}^{\mathrm{III}}$ ions as a central building block, namely $\left[\mathrm{Dy}_{3}\left(\mu_{3}-\mathrm{OH}\right)_{2} \mathrm{~L}_{3} \mathrm{Cl}_{2}\left(\mathrm{H}_{2} \mathrm{O}\right)_{4}\right]-$ $\left[\mathrm{Dy}_{3}\left(\mu_{3}-\mathrm{OH}\right)_{2} \mathrm{~L}_{3} \mathrm{Cl}\left(\mathrm{H}_{2} \mathrm{O}\right)_{5}\right] \mathrm{Cl}_{5} \cdot 19 \mathrm{H}_{2} \mathrm{O}(\mathbf{1} ; \mathrm{HL}=o$-vanillin $),{ }^{[19]}$ $\left[\mathrm{Dy}_{3}\left(\mu_{3}-\mathrm{OH}\right)_{2} \mathrm{~L}_{3} \mathrm{Cl}\left(\mathrm{H}_{2} \mathrm{O}\right)_{5}\right] \mathrm{Cl}_{3} \cdot 4 \mathrm{H}_{2} \mathrm{O}$ (2), ${ }^{[19]}$ and $\left[\mathrm{Dy}_{3} \mathrm{Cu}_{6} \mathrm{~L}^{1}{ }^{-}\right.$

[*] Prof. L. F. Chibotaru, L. Ungur, Dr. A. Soncini

Division of Quantum and Physical Chemistry

Department of Chemistry

Katholieke Universiteit Leuven

Celestijnenlaan 200F, 3001 Leuven (Belgium)

Fax: $(+32)$ 1632-7992

E-mail: liviu.chibotaru@chem.kuleuven.be

[***: Financial support by the Flemish National Science Foundation and the Flemish Government under the concerted action scheme is gratefully acknowledged. The authors thank Prof. Annie Powell, Karlsruhe and Prof. Roberta Sessoli, Florence for useful discussions and for providing them with digitalized magnetic data.

$\square$ Supporting information for this article is available on the WWWW under http://www.angewandte.org or from the author.
$\left.\left(\mu_{3}-\mathrm{OH}\right)_{6}\left(\mathrm{H}_{2} \mathrm{O}\right)_{10}\right] \mathrm{Cl}_{2} \cdot \mathrm{ClO}_{4} \cdot 3.5 \mathrm{H}_{2} \mathrm{O}\left(\mathbf{3} ; \mathrm{H}_{2} \mathrm{~L}^{1}=1,1,1\right.$-trifluoro7-hydroxy-4-methyl-5-azahept-3-en-2-one), ${ }^{[20]}$ have recently been synthesized and investigated. All these compounds show slow relaxation of magnetization. Compound $\mathbf{3}$ is characterized by a relatively high anisotropy barrier of $25 \mathrm{~K}$, whereas 1 and $\mathbf{2}$ show a vanishingly small susceptibility at low temperature, which was completely unexpected for systems containing an odd number of electrons. Both these latter compounds show similar magnetic properties despite the presence of very different magnetic networks, ${ }^{[19]}$ which allows intermolecular antiferromagnetic exchange interactions to be ruled out as a reason for this vanishingly small susceptibility at low temperature. In view of such an unprecedented situation, we have investigated the local anisotropy of the dysprosium sites in these complexes by high-level ab initio calculations and have simulated, on their basis, the lowest exchange states. This procedure has allowed an unambiguous determination of the nature of the ground state in these complexes.

The main structural difference between the triangular units in $\mathbf{1}$ and $\mathbf{2}$ (Figure 1) is the $\operatorname{Dy}(3)$ site, which is coordinated by one chloride ion above and one water molecule below the plane of the triangle in $\mathbf{1}$ whilst in $\mathbf{2}$ this site is occupied by a chloride ion above the plane and a chloride ion or a water molecule below the plane with a 50:50 disorder. Since the magnetic data for $\mathbf{1}$ and $\mathbf{2}$ are almost

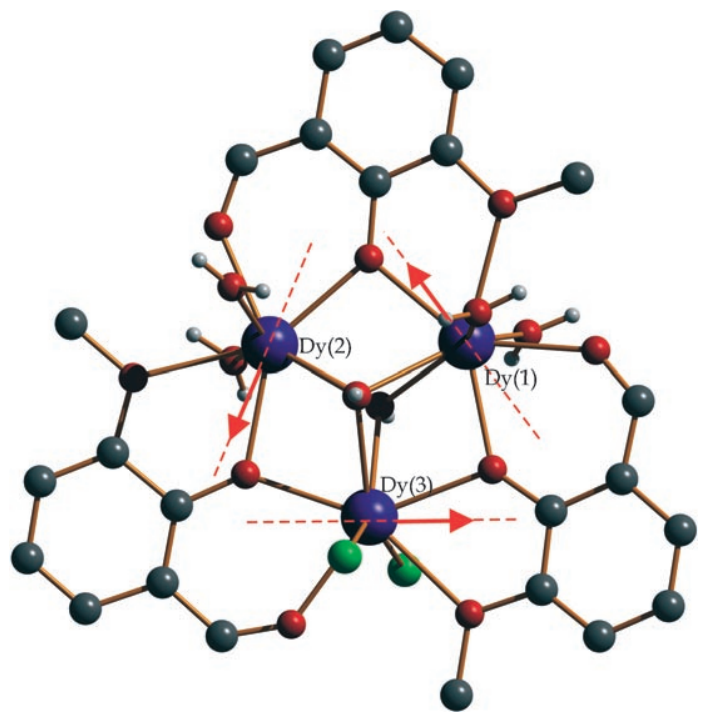

Figure 1. Structure of the triangular units in 1 and $2 .^{[19]}$ The structure with two chloride ions coordinated to Dy(3) was used in the ab initio calculations. Color scheme: blue $\mathrm{Dy}^{\prime \prime \prime}$; red $\mathrm{O}$; green $\mathrm{Cl}$; dark grey $\mathrm{C}$; white $\mathrm{H}$. The dashed lines show the calculated anisotropy axes on the dysprosium fragments and the arrows show the ordering of local magnetizations in the ground state of the complex. 
identical, ${ }^{[19]}$ we can conclude that substitution of the water molecule by a chloride ion on the Dy(3) site does not change the magnetic properties of the $\mathrm{Dy}_{3}$ unit, therefore below we consider the complex with the $\mathrm{Dy}(3)$ unit coordinated by two chloride ions (Figure 1). We performed complete active space self-consistent-field (CASSCF) calculations for each dysprosium fragment of this unit and included the effects of spinorbit coupling by mixing all terms with energies lower than $50000 \mathrm{~cm}^{-1}$ (see the Supporting Information for computational details). As expected, we obtained a group of eight Kramers doublets, originating mainly from the ground ${ }^{6} H_{15 / 2}$ multiplet of the $\mathrm{Dy}^{3+}$ ion (Table 1), for the lowest states of each dysprosium site, which are separated from the other excited states by around $3000 \mathrm{~cm}^{-1}$. As shown in Table 1 , the ground Kramers doublet is separated from the rest by a relatively large gap $\left(>200 \mathrm{~cm}^{-1}\right)$.

Table 1: Lowest calculated Kramers doublets (KDs) of the three dysprosium fragments of $\mathbf{1}$.

\begin{tabular}{llll}
\hline KD & $\begin{array}{l}\text { Dy }(1) \\
\text { Energies }\left[\mathrm{cm}^{-1}\right]\end{array}$ & Dy(2) & Dy(3) \\
\hline 1 & 0.0 & 0.0 & 0.0 \\
2 & 234.2 & 217.8 & 150.2 \\
3 & 373.3 & 367.0 & 208.1 \\
4 & 449.0 & 479.3 & 258.2 \\
5 & 501.2 & 532.0 & 336.8 \\
6 & 554.4 & 638.1 & 435.6 \\
7 & 657.9 & 684.5 & 579.9 \\
8 & 741.4 & 763.0 & 707.5 \\
& & & \\
& Main values of the g tensor & 0.064 \\
& 0.003 & 0.003 & 0.089 \\
& 0.005 & 0.004 & 19.740 \\
1 & 19.844 & 19.837 & \\
& Angle of anisotropy axis with Dy ${ }_{3}$ plane & \\
& $-4.3^{\circ}$ & $8.8^{\circ}$ & $-2.4^{\circ}$ \\
\hline
\end{tabular}

Using a recently developed ab initio methodology, ${ }^{[21]}$ we calculated the $g$ tensors of the three dysprosium fragments in the ground Kramers doublet. The main values of the $g$ tensors, which correspond to an effective spin $\tilde{S}=1 / 2$ of the Kramers doublet, are listed in Table 1. We can see that the tensors obtained are very anisotropic and closely resemble the tensors of the Kramers doublet of a pure $\left|M_{\mathrm{J}}= \pm 15 / 2\right\rangle$ type $^{[22]}$ despite the lack of axial symmetry on the sites (Figure 1). The calculated directions of the anisotropy axes at the three dysprosium sites are shown in Figure 1 by dashed lines. The three axes form an almost perfect equilateral triangle and lie practically in the $\mathrm{Dy}_{3}$ plane (the deviation angles are listed in Table 1 ).

We also calculated the magnetic susceptibility of the individual dysprosium fragments using the same ab initio approach (Figure 2, dashed line). ${ }^{[21]}$ The exchange interaction between dysprosium sites was simulated within the Lines model, ${ }^{[23]}$ in which an effective Heisenberg Hamiltonian, $H_{\mathrm{ex}}=-\sum_{i=1}^{3} J_{i} \mathbf{S}_{i} \cdot \boldsymbol{S}_{i+1}$, which corresponds to local $S=5 / 2$ spins on dysprosium sites in the absence of spin-orbit coupling, was diagonalized on the basis of the Kramers doublets obtained

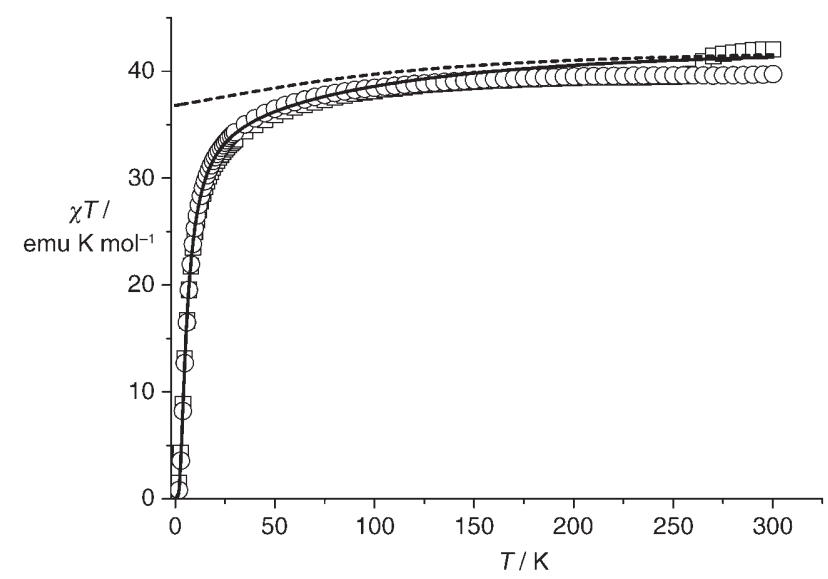

Figure 2. Temperature dependence of $\chi T$ per $\mathrm{Dy}_{3}$ molecule calculated as a sum of independent dysprosium fragments (dashed line) and simulated for $J=-0.6 \mathrm{~cm}^{-1}$ (line) for a polycrystalline sample and the experimental powder; empty squares: data for 1 ; empty circles: data for 2 .

from fragment ab initio calculations. ${ }^{[21]}$ This approach is appropriate in this case because the local Kramers doublets are found to be close to $\left|M_{\mathrm{J}}= \pm 15 / 2\right\rangle$. In this case, given the smallness of the exchange interactions, we expect the exchange interaction to be of the Ising type [Eq (1)]. In this

$\tilde{H}_{\mathrm{ex}}=-\sum_{i=1}^{3} \tilde{J}_{i} \tilde{S}_{i z} \tilde{S}_{i+1 z}$

equation, $\tilde{S}_{i z}$ is the projection of the pseudo-spin on the anisotropy axis of site $i$ (Figure 1) and describes the two states with (opposite) maximal magnetization on this site. Within the Lines model we come to the same Ising interaction as that in Equation (1) with $\tilde{J}_{\mathrm{i}}=25 \cos \varphi_{i, i+1} J_{i}$, where $\varphi_{i, i+1}$ is the angle between the anisotropy axes on sites $i$ and $i+1$. Since $\varphi_{i, i+1}$ $\approx 2 \pi / 3$ (Figure 1 ), $\tilde{J}_{\mathrm{i}} \approx-12.5 J_{i}$. Given the magnetic similarity of the dysprosium fragments obtained from ab initio calculations, we suppose further that $J_{\mathrm{i}}=J\left(\tilde{J}_{\mathrm{i}}=\tilde{J}\right)$.

The simulated powder magnetic susceptibility and magnetization data for $J=-0.6 \mathrm{~cm}^{-1}$ (the only fitting parameter of the theory) are shown in Figures 2 and 3, respectively, where it can be seen that $\chi T$ almost vanishes at low temperature, which means an almost nonmagnetic ground state. The origin of this state is easy to understand as $\tilde{J}>0$ for antiferromagnetic $J$ and Equation (1) therefore predicts a ferromagnetic alignment of the pseudospins (and local magnetization vectors) along the anisotropy axes, as shown by the arrows in Figure 1. Since the local magnetization vectors are almost tangential to the vertices of the $\mathrm{Dy}_{3}$ triangle, we have here an example of an almost perfectly toroidal magnetic moment. The second component of the Kramers doublet is obtained by a time-inversion operation which changes the directions of all magnetic moments (Scheme 1).

The inset in Figure 3 shows that the magnetization is not zero, even at very low temperature, but increases with the field and comes almost to saturation before rising steeply at an $H$ value of around $0.8 \mathrm{~T}$; this points to a remnant small 


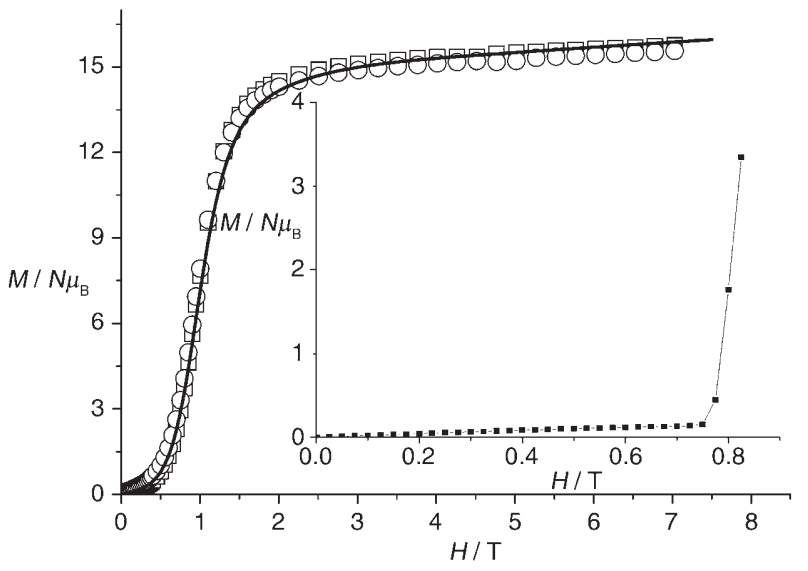

Figure 3. Molar magnetization $M$ versus the applied magnetic field $H$ calculated for $J=-0.6 \mathrm{~cm}^{-1}$ (lines) for a polycrystalline sample and the experimental powder magnetization for $\mathbf{1}$ (empty squares) and $\mathbf{2}$ (empty circles) for $T=1.8 \mathrm{~K}$. Inset: the same simulations for $T=0.1 \mathrm{~K}$.
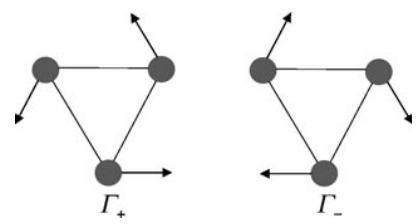

Scheme 1. The two components of the ground Kramers doublet in 1 and 2 . The arrows show the direction of magnetization on the dysprosium sites.

magnetic moment in the ground state. Figure 4 shows the evolution of four exchange Kramers doublets of $\mathrm{Dy}_{3}$, which arise from the exchange interaction between the lowest Kramers doublets on dysprosium sites, in a magnetic field. The ground Kramers doublet $(\mathrm{KD}=0)$ splits almost linearly with the field applied perpendicular to the plane (Figure $4 \mathrm{a}$ ) and shows no splitting for the in-plane direction of the field (Figure 4b), which means that the noncompensated magnetic moment is directed perpendicular to the plane. Accordingly, the $g$ tensor calculated for the ground Kramers doublet shows $g_{1}=0.56$ and $g_{2}=g_{3}=0$, with the main magnetic axis directed perpendicular to the $\mathrm{Dy}_{3}$ plane. The lowest excited Kramers doublets correspond to reversal of the direction of magnetization on one of the three dysprosium sites (Scheme 1). According to Equation (1) their excitation energies are approximately $-\tilde{J}=7.5 \mathrm{~cm}^{-1}$, which is indeed observed (Figure 4, energy levels at zero field). In addition, the complex acquires a magnetic moment of around twice the moment of an individual dysprosium site (approx. $19.7 \mu_{\mathrm{B}}$ ) in each of these states. As a result, a steep rise of magnetization occurs close to the crossing fields. As Figure 4 shows, these crossing fields differ several times for the in-plane and perpendicular to the plane directions, which means that the $\mathrm{Dy}_{3}$ plane is the easy plane of magnetization.

In summary, we have provided evidence that the origin of the nonmagnetic ground state in dysprosium(III) triangles $\mathbf{1}$ and $\mathbf{2}$ is the toroidal arrangement of magnetic moments on the dysprosium sites. To the best of our knowledge, this is the first time that an almost perfect toroidal magnetic moment has
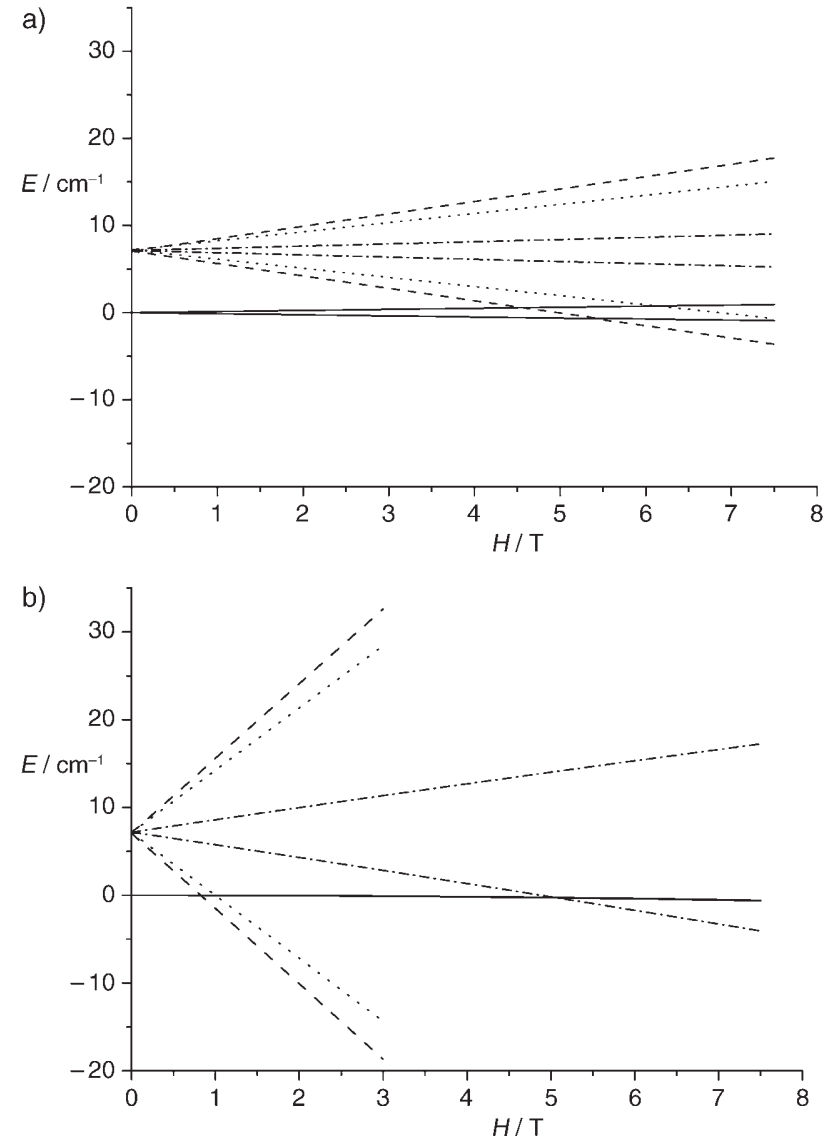

Figure 4. Evolution of the lowest magnetic states of a $\mathrm{Dy}_{3}$ complex with applied magnetic field, simulated for $J=-0.6 \mathrm{~cm}^{-1}$. a) $H$ is perpendicular to the $\mathrm{Dy}_{3}$ plane. b) $\mathrm{H}$ is applied in the $\mathrm{Dy}_{3}$ plane.

been detected in a molecular magnet. This toroidal moment does not interact with a homogeneous dc magnetic field but otherwise it has many features in common with a dipolar magnetic moment. In particular, it is characterized by two directions of toroidal magnetization (shown in Scheme 1). The ground Kramers doublets on the dysprosium sites support an Ising exchange interaction, thereby preserving the states of maximal magnetization on these sites. This means that reorientation of the toroidal magnetization requires consecutive transitions through three excited Kramers doublets of $\mathrm{Dy}_{3}$ whose excitation energies represent the barrier of blockage of this magnetization. ${ }^{[24]}$

Received: January 19, 2008

Published online: April 21, 2008

Keywords: ab initio calculations - dysprosium . exchange coupling $\cdot$ magnetic properties . single-molecule magnets

[1] A. Caneschi, D. Gatteschi, R. Sessoli, A. L. Barra, L. C. Brunel, M. Guillot, J. Am. Chem. Soc. 1991, 113, 5873-5874.

[2] P. D. W. Boyd, Q. Y. Li, J. B. Vincent, H. R. Chang, W. E. Streib, J. C. Huffman, G. Christou, D. N. Hendrickson, J. Am. Chem. Soc. 1988, 110, 5537-5539. 
[3] R. Sessoli, D. Gatteschi, A. Caneschi, M. Novak, Nature 1993 $365,141-143$.

[4] R. Sessoli, H. L. Tsai, A. R. Schake, S. Wang, J. B. Vincent, K. Folting, D. Gatteschi, G. Christou, D. Hendrickson, J. Am. Chem. Soc. 1993, 115, 1804-1816.

[5] D. Gatteschi, R. Sessoli, Angew. Chem. 2003, 115, 278-309; Angew. Chem. Int. Ed. 2003, 42, 268-297.

[6] G. Christou, D. Gatteschi, D. Hendrickson, R. Sessoli, MRS Bull. 2000, $25,66-71$.

[7] D. D. Awschalom, D. P. Di Vincenzo, Phys. Today 1995, 48, $43-$ 48.

[8] E. M. Chudnovsky, Science 1996, 274, 938-939.

[9] M. N. Leuenberger, D. Loss, Nature 2001, 410, 789-793.

[10] M. Affronte, F. Troiani, A. Ghirri, A. Candini, M. Evangelisti, V. Corradini, S. Carretta, P. Santini, G. Amoretti, F. Tuna, G. Timco, R. E. P. Winpenny, J. Phys. D 2007, 40, 2999-3004.

[11] A. Bencini, D. Gatteschi, Electron Paramagnetic Resonance of Exchange Coupled Systems, Springer, Berlin, 1990, chap. 3.

[12] L. G. Westin, M. Kritikos, A. Caneschi, Chem. Commun. 2003, $1012-1013$.

[13] N. Ishikawa, M. Sugita, W. Wernsdorfer, J. Am. Chem. Soc. 2005, $127,3650-3651$.

[14] S. Osa, T. Kido, N. Matsumoto, N. Re, A. Pochaba, J. Mrozinski, J. Am. Chem. Soc. 2004, 126, 420-421.
[15] C. M. Zaleski, E. C. Depperman, J. W. Kampf, M. L. Kirk, V. L. Pecoraro, Angew. Chem. 2004, 116, 4002-4004; Angew. Chem. Int. Ed. 2004, 43, 3912-3914.

[16] A. Mishra, W. Wernsdorfer, K. A. Abboud, G. Christou, J. Am. Chem. Soc. 2004, 126, 15648-15649.

[17] A. Mishra, W. Wernsdorfer, S. Parsons, G. Christou, E. K. Brechin, Chem. Commun. 2005, 2086-2088.

[18] V. S. Mironov, L. F. Chibotaru, A. Ceulemans, J. Am. Chem. Soc. 2003, 125, 9750-9760.

[19] J. Tang, I. Hewitt, N. T. Madhu, G. Chastanet, W. Wernsdorfer, C. E. Anson, C. Benelli, R. Sessoli, A. K. Powell, Angew. Chem. 2006, 118, 1761-1765; Angew. Chem. Int. Ed. 2006, 45, 17291733.

[20] J. C. Aronica, G. Pillet, G. Chastanet, W. Wernsdorfer, J.-F. Jacquot, D. Luneau, Angew. Chem. 2006, 118, 4775-4778; Angew. Chem. Int. Ed. 2006, 45, 4659-4662.

[21] L. F. Chibotaru, L. Ungur, unpublished results.

[22] J. Hog, P. Touborg, Phys. Rev. B 1975, 11, 520-529.

[23] M. E. Lines, J. Chem. Phys. 1971, 55, 2977-2984.

[24] Note added in proof: After this article was submitted, we learned about additional magnetic measurements on the Dy(III) triangles which were interpreted within a non-collinear Ising model, also resulting in a non-magnetic ground state: J. Luzon, K. Bernot, I. J. Hewitt, C. E. Anson, A. K. Powell, R. Sessoli, Phys. Rev. Lett. cond-mat:0804.1272v1; in press. 